\title{
Avaliação do Conhecimento e da Conduta de Urgência Pós-Traumatismo Dentário
}

\author{
Gabriel dos Santos Marques, ${ }^{1}$ Jean Alves dos Santos, ${ }^{1}$ Sara Sabrina de Sena, ${ }^{1}$ \\ Nathália Evelyn da Silva Machado, ${ }^{2}$ Rosana Leal do Prado, ${ }^{1}$ \\ Juliane Avansini Marsicano, ${ }^{1}$ Graziela Garrido Mori ${ }^{3}$
}

\begin{abstract}
RESUMO
Traumatismos dentários são muito comuns em razão de quedas e acidentes, afetando, principalmente, crianças em idade escolar. $\mathrm{O}$ adequado atendimento de urgência para estes casos é fundamental para o estabelecimento da saúde bucal, e qualquer pessoa, presente no local do acidente, pode colaborar decisivamente com o prognóstico do caso. Assim, o presente estudo teve como objetivo avaliar as condutas de professores e funcionários de escolas públicas ou privadas que trabalham com crianças entre 7 e 11 anos ante o traumatismo e a avulsão dentária. Inicialmente, aplicou-se o questionário proposto como ferramenta de avaliação para 50 professores e funcionários de escolas, com o objetivo de validação de tal ferramenta. Após coleta e análise dos questionários respondidos, realizaram-se pequenas alterações e nova aplicação do questionário validado. Nesta segunda etapa foram incluídos 231 professores e funcionários de escolas da rede pública e privada que trabalham com crianças de 7 a 11 anos. Os dados foram tabulados e analisados estatisticamente por meio do teste do Qui-quadrado e teste $\mathrm{G}(\mathrm{p}<0.05)$. Somente $32,9 \%$ dos participantes levariam o acidentado imediatamente para o dentista. Cerca de $26 \%$ dos participantes já tiveram experiência pessoal com traumatismo dentário, e $10 \%$ sofreram avulsão dentária. Quando perguntados sobre o reimplante imediato, somente $14,7 \%$ o faria e apenas $20,3 \%$ fariam a limpeza correta do dente. O leite como meio de conservação adequado representou $16 \%$ das respostas. Os professores e funcionários de escolas públicas e privadas apresentam baixo conhecimento sobre traumatismo dentário, e as condutas desta população podem ser aprimoradas por meio de campanhas de esclarecimento.

Palavras-chave: Traumatismos dentários. Avulsão dentária. Conhecimento. Reimplante dentário.
\end{abstract}

EVALUATION OF KNOWLEDGE AND EMERGENCY MANAGEMENT AFTER DENTAL TRAUMA

\section{ABSTRACT}

Dental injuries are very common due to falls and accidents, affecting mainly school-age children. Adequate emergency care for these cases is essential for the establishment of oral health and anyone present at the scene of the accident can contribute decisively to the prognosis of the case. Thus, the present study aimed to evaluate the conduct of teachers and employees of public or private schools working with children between age 7 and 11 years, before trauma and dental avulsion. Initially, the proposed questionnaire was applied as an evaluation tool for 50 teachers and school workers aiming to validate it. After collecting and analyzing the questionnaires answered, minor changes were made and the validated questionnaire was re-applied. In this second stage were included 231 teachers and employees of public and private schools, working with children from 7 to 11 years. Data were tabulated and statistically analyzed using Chi-squared test and $\mathrm{G}$ test $(p<0.05)$. Only $32.9 \%$ of participants would take the injured person immediately to the dentist. About $26 \%$ of participants have already had personal experience with dental trauma, and $10 \%$ have had dental avulsion. When asked about immediate replantation, only $14.7 \%$ would do so and only $20.3 \%$ would clean the tooth correctly. Milk as a suitable storage medium represented $16 \%$ of the responses. Teachers and employees of public and private schools showed low knowledge about dental trauma and this population management can be improved through information campaigns.

Keywords: Tooth injuries. Tooth avulsion. Knowledge. Tooth replantation.

RECEBIDO EM: 20/11/2019

ACEITO EM: $12 / 8 / 2020$

${ }^{1}$ Universidade do Oeste Paulista - Unoeste. Presidente Prudente/SP, Brasil.

Universidade Estadual Paulista - Unesp. Araçatuba/SP, Brasil.

${ }^{3}$ Autora correspondente. Universidade do Oeste Paulista - Unoeste. Rua José Bongiovani, 700 - Cidade Universitária. Presidente Prudente/SP, Brasil. CEP 19050-920. http://lattes.cnpq.br/9461725931162862. http://orcid.org/0000-0002-8690-5294. grazielagm@hotmail.com 


\section{INTRODUÇÃO}

Sabe-se que traumatismos dentários envolvendo crianças e adultos são frequentes (ANDREASEN; ANDREASEN; ANDERSSON, 2018). Estes correspondem a lesões que vão desde uma simples fratura em esmalte até a perda temporária do elemento dentário, podendo ser classificadas em fraturas coronárias, radiculares, coronoradiculares e injúrias por luxação, que incluem concussão, subluxação, luxação lateral, intrusiva e extrusiva, e avulsão dentária, estando associadas a acidentes e quedas principalmente (ANDREASEN; ANDREASEN; ANDERSSON, 2018; ANDERSSON et al., 2012).

A avulsão dentária refere-se ao completo deslocamento do dente para fora de seu alvéolo de origem (ANDREASEN; ANDREASEN; ANDERSSON, 2018; ANDERSSON et al., 2012). Segundo Andreasen et al. (2012), a avulsão dentária é a lesão traumática mais complexa, e o sucesso do seu tratamento depende das ações tomadas no local do acidente. A avulsão dentária representa aproximadamente $15 \%$ das lesões traumáticas da dentição permanente, ocorrendo, com mais frequência, em pacientes de 7 a 11 anos de idade (ANDREASEN; ANDREASEN; ANDERSSON, 2018; ANDERSSON et al., 2012).

$O$ atendimento de urgência para dentes traumatizados é fundamental para o sucesso do tratamento (ANDREASEN; ANDREASEN; ANDERSSON, 2018; ANDERSSON et al., 2012; SANABE et al., 2009). Em casos de avulsão dentária, o ideal é que o dente seja reimplantado o mais rápido possível, entre 15 e $30 \mathrm{mi}$ nutos (ANDREASEN; ANDREASEN; ANDERSSON, 2018; ANDERSSON et al., 2012; TROPE, et al., 2011). O reimplante dentário imediato pode evitar a necrose das células do ligamento periodontal e da polpa dentária, além de limitar os processos inflamatório e infeccioso, que influenciarão na ocorrência da anquilose e da reabsorção dentária, alterações que podem levar à perda do dente (ANDREASEN; ANDREASEN; ANDERSSON, 2018; ANDERSSON et al., 2012; YAMASHITA, et al., 2017).

Caso o reimplante dentário imediato não seja possível, o dente avulsionado pode ser armazenado em um meio de conservação; este deve conter características antimicrobianas, ser capaz de manter a viabilidade das células do ligamento periodontal por um período de tempo aceitável, favorecer a capacidade proliferativa celular, bem como possuir a mesma osmolaridade das células do ligamento periodontal e da polpa dentária (em torno de $230 \mathrm{mOsm} / \mathrm{kg}$ ) (ANDREASEN; ANDREASEN; ANDERSSON, 2018; ANDERSSON et al., 2012; FLORES et al., 2016; MORI et al., 2010). Dentre os meios recomendados pode-se citar: Solução Salina Balanceada de Hank's (HBSS), leite, solução salina e saliva (ANDREASEN; ANDREASEN; ANDERSSON, 2018; ANDERSSON et al., 2012; TROPE, et al., 2011; FLORES et al., 2016; MORI et al., 2010).

A HBSS é uma composição biocompatível, sendo capaz de preservar e estimular a proliferação das células do ligamento periodontal por até 24 horas (KHINDA, et al., 2017; TROPE; FRIEDMAN, 1992). Esta, no entanto, não é disponível no Brasil. O leite é um meio de armazenamento adequado por suas propriedades fisiológicas, incluindo $\mathrm{pH}$ e osmolaridade ideal, além de possuir fator de crescimento epitelial (EGF) que mantém a vitalidade das células do ligamento periodontal (MORI et al., 2010; KHINDA, et al., 2017; REIS et al., 2017). Além de estimular a proliferação celular, ele pode prolongar o tempo de reimplante por até 6 horas (MORI et al., 2010). Solução salina e saliva não são ideais, mas podem ser uma opção ao meio seco quando o leite não estiver disponível, não devendo exceder 60 minutos (ANDREASEN; ANDREASEN; ANDERSSON, 2018; ANDERSSON et al., 2012; KHINDA, et al., 2017; FLORES et al., 2016).

Estas condutas são adequadas ante a casos de avulsão dentária, e o conhecimento sobre estas é crucial para o sucesso do reimplante dentário, que culminará na manutenção funcional e fonética do dente, além de preservar a estética e não promover um impacto psicológico negativo ao paciente (ANDREASEN; ANDREASEN; ANDERSSON, 2018).

Nesse contexto, deve-se ressaltar que qualquer pessoa que esteja próxima a alguém que sofreu traumatismo dentário é capaz de realizar o atendimento de urgência, seja realizando o reimplante dentário imediato, seja armazenando o dente em meio de conservação adequado, como citado anteriormente (ANDREASEN; ANDREASEN; ANDERSSON, 2018; ANDERSSON et al., 2012). O atendimento de urgência conclui-se com o ato de levar o acidentado ao cirugião dentista o mais rápido possível (ANDREASEN; ANDREASEN; ANDERSSON, 2018; ANDERSSON et al., 2012; MORI et al., 2009), e é importante salientar que situações como estas são difíceis tanto para os acidentados quanto para os responsáveis ou pessoas próximas, que terão de lidar com os primeiros socorros (ANDREASEN; ANDREASEN; ANDERSSON, 2018).

Crianças com idade média de 7 a 11 anos são extremamente susceptíveis ao traumatismo dentário, e acidentes envolvendo-as podem ocorrer no ambiente escolar (ANDREASEN; ANDREASEN; ANDERSSON, 2018; ANDERSSON et al., 2012). Assim, justifica-se a 
opção pela análise da conduta de urgência dos professores e funcionários do Ensino Fundamental quanto ao traumatismo dentário, com ênfase na avulsão dentária, posto que, muitas vezes, estes são os primeiros a tomar medidas imediatas, interferindo decisivamente no prognóstico do reimplante dentário.

Considerando os aspectos abordados supra e a importância da promoção da saúde bucal, o presente estudo teve como objetivo avaliar o conhecimento sobre traumatismo dentário e sua prevenção, bem como analisar as condutas, neste caso, de professores e funcionários de escolas públicas ou privadas que trabaIham com crianças entre 7 e 11 anos.

\section{MATERIAL E MÉTODOS}

O presente trabalho foi realizado somente após a aprovação do Comitê de Ética em Pesquisa (CAAE:63031116.8.0000.5515). Para a realização deste, os pesquisadores aplicaram um questionário específico a professores e funcionários de escolas públicas e privadas do Ensino Fundamental que trabalham com crianças entre 7 e 11 anos. Todos os participantes da pesquisa assinaram o Termo de Consentimento Livre e Esclarecido.

A partir dos questionários publicados nos trabalhos de Mori et al. $(2005,2009)$, um novo questionário foi confeccionado, abrangendo perguntas sobre conhecimentos gerais e prevenção do traumatismo dentário, além do enfoque específico nas condutas de urgência relacionadas com a avulsão e o reimplante dentários. O questionário foi dividido em três partes: na parte I havia questões sobre idade, sexo, nível educacional e o tempo de trabalho em instituições de ensino dos participantes da pesquisa; na parte II questões sobre experiência anterior com traumatismo dentário, formas de prevenção e procedimentos de urgência; e na parte III perguntas específicas sobre avulsão e reimplante dentário.

Este questionário foi aplicado, inicialmente, para um grupo de 50 professores e funcionários de escolas de Ensino Fundamental da cidade de Presidente Prudente, São Paulo. Para isso, os questionários foram entregues aos participantes da pesquisa e os mesmos, sem necessidade de identificação, tiveram um período, previamente combinado com a escola, para as respostas. Os questionários foram recolhidos e os dados tabulados e analisados pelo programa Epilnfo para sua validação. Correções foram realizadas em questões com dificuldade de resposta em razão da ausência de compreensão da pergunta, estabelecendo-se um novo questionário válido com as seguintes mudanças: (1) acréscimo de orientação quanto a escolher somente uma opção por questão, posto que alguns entrevistados assinalavam várias opções; (2) na parte II do questionário houve a alteração da posição da questão 5 , além do acréscimo de orientações, pois, da forma original, muitas dúvidas foram levantadas; (3) simplificação da questão 2 da parte III (Figura 1 Questionário Validado).

Após a validação do questionário, o mesmo foi aplicado para um grupo de 231 professores e funcionários de escolas públicas e privadas de Ensino Fundamental da citada cidade. Os participantes tiveram um intervalo de tempo que foi determinado pelos responsáveis das escolas de acordo com as atividades da mesma; em algumas escolas a resposta foi imediata, e, em outras, de 4 a 7 dias para a coleta dos questionários respondidos.

Os dados foram tabulados em uma planilha do Excel e com o auxílio do programa estatístico R Studio. Os resultados foram estabelecidos, definindo a porcentagem das respostas de acordo com as questões e partes do questionário. Além disso, com o auxílio dos testes do Qui-quadrado e teste $\mathrm{G}$, pode-se determinar se experiência prévia com o traumatismo dentário, idade, tempo de trabalho em escola e escolaridade poderiam influenciar no grau de conhecimento e na conduta dos professores e funcionários de escolas. 0 grau de significância utilizado foi de $5 \%(p<0.05)$.

\section{RESULTADOS}

Um total de 231 questionários foi respondido, dos quais $118(51,1 \%)$ foram de escolas públicas e $113(48,9 \%)$ de escolas privadas. A primeira parte do questionário tratava sobre os dados gerais dos participantes. Em relação ao sexo, o masculino representou $12,1 \%$ dos entrevistados, e o sexo feminino, $87,9 \%$. Em relação à idade, 1 participante declarou possuir 18 anos ( $0,4 \%$ do total); 107 declararam possuir idade entre 19 e 40 anos (46,3\% do total); 108 entrevistados tinham entre 41 e 60 anos $(46,8 \%)$, e 15 participantes $(6,5 \%)$ tinham 61 anos ou mais.

Dos professores e funcionários avaliados, pode-se verificar que $11,3 \%$ estudaram até o Ensino Fundamental, $15,2 \%$ concluíram o Ensino Médio e $69,3 \%$ o Ensino Superior. Um total de 4,3\% dos entrevistados não informou este dado. Cerca de $16 \%$ dos participantes da pesquisa afirmaram que trabalharam na instituição por até 1 ano, 33,8\% de 1 a 5 anos, $13 \% 6$ a 10 anos, $11,7 \%$ de 11 a 15 anos e $24,7 \%$ com tempo igual ou superior a 16 anos. Duas pessoas não informaram $(0,9 \%)$. 
A segunda parte do questionário avaliou a conduta inicial e geral nos casos de traumatismo dentário e também sobre as experiências anteriores dos professores e funcionários. Quando perguntados sobre o que fariam se presenciassem algum tipo de traumatismo dentário, a maioria respondeu que acalmaria o acidentado e procuraria um pronto-socorro $(49,4 \%$ dos participantes), 32,9\% levaria o acidentado imediatamente para o dentista, $3 \%$ procuraria o hospital, $0,9 \%$ procuraria um médico, $1,7 \%$ não informou e $3 \%$ não saberiam como proceder nesses casos. Cerca de $9 \%$ dos participantes descreveram outras opções, como avisar a direção da escola ou ligar para os pais dos alunos.

Um total de $26 \%$ dos entrevistados já foram acometidos por algum tipo de traumatismo dentário. Os tipos ocorridos e seus índices podem ser observados na Figura 2.

Entre os participantes com experiência pessoal de traumatismo dentário, $40 \%$ procuraram um dentista imediatamente e $28,8 \%$ procuraram um dentista no dia seguinte; $10 \%$ procuraram um pronto-socorro, $10 \%$ não fizeram nada, $1,67 \%$ não responderam e $10 \%$ indicaram outro procedimento, com somente 1 esclarecendo que procurou um médico. Ao serem perguntado sobre a presença de algum tipo de sequela, $31,67 \%$ relataram ausência, $28,33 \%$ disseram que tiveram sequelas, mas que foram submetidos a tratamento odontológico e o problema foi resolvido, $20 \%$ relataram que houve a necessidade de exodontia dentária, $10 \%$ descreveram alteração de cor da coroa do dente envolvido, $1,67 \%$ não responderam e $8,33 \%$ indicaram outras sequelas, e somente 1 esclareceu que foi a giro-versão dentária.

Ao correlacionar a experiência pessoal de traumatismo dentário com a conduta inicial, caso presenciasse algum tipo de acidente envolvendo os dentes ou a face, pode-se verificar que aquela não influencia nesta. A conduta inicial ante o tratamento dentário, de modo geral, foi similar entre os entrevistados que sofreram traumatismo e os que não sofreram ( $p=0.8953)$.

A terceira parte do questionário proporcionou a investigação sobre as condutas relacionadas especificamente com a avulsão dentária. Cerca de $10 \%$ dos participantes já tiveram experiência com avulsão dentária, e aproximadamente $91 \%$ acreditam que alguma conduta deva ser realizada com urgências nesses casos.

Somente $14,7 \%$ dos participantes teriam a iniciativa de realizar o reimplante imediato, demonstrando baixos índices de conhecimento em relação à importância daquele. Quando perguntados sobre se achavam necessário limpar o dente antes do reimplante, 8 (3,5\%) participantes não responderam e 205 $(88,7 \%)$ acreditam que ser necessário. As condutas relacionadas a como fariam a limpeza do dente antes do reimplante, podem ser observadas na Figura 3.

Caso não reimplantassem o dente avulsionado, $97 \%$ dos pacientes transportariam o dente para o dentista nos meios de conservação especificados na Figura 4.

A experiência prévia com traumatismo dentário e/ou avulsão dentária não interferiu na conduta do participante em relação à avulsão, seja na realização do reimplante imediato, seja na utilização do meio de conservação adequado $(p>0,05)$.

Ao correlacionar os dados pessoais com as condutas em relação ao traumatismo dentário e/ou avulsão dentária, pode-se verificar que a escolaridade não interferiu na conduta dos participantes $(p>0,05)$. 0 tempo de trabalho na escola, no entanto, favoreceu o uso correto do meio de conservação para dentes avulsionados ( $p=0.02701$ ), posto que os participantes com 16 anos ou mais de trabalho apresentaram conhecimento favorável em relação a este aspecto.

Não houve diferença entre as respostas dos participantes de escolas públicas e escolas privadas $(p>0,05)$.

\section{DISCUSSÃO}

O traumatismo dentário representa um significante problema de saúde pública (GLENDOR, 2008; MAGNO et al., 2019), podendo envolver problemas estéticos, fonéticos, funcionais e psicossocial, afetando, consequentemente, a qualidade de vida dos envolvidos (ANDREASEN; ANDREASEN; ANDERSSON, 2018). A análise do conhecimento para a conduta de urgência pelos professores e funcionários de escolas que trabalham com crianças entre 7 a 11 anos, que é uma das populações mais acometidas (ANDREASEN; ANDREASEN; ANDERSSON, 2018; ANDERSSON et al., 2012), portanto, é fundamental para o estabelecimento de medidas preventivas e de orientação a esta população, com o objetivo primordial de diminuir as perdas dentárias em consequência dos traumatismos.

A referida análise também foi alvo de outros autores, confirmando a importância do presente estudo (CHANDUKUTTY et al., 2017; MORI et al., 2005; CALDERA et al., 2018). Assim como os resultados presentes neste trabalho, os citados autores também verificaram reduzido conhecimento adequado sobre 
condutas de urgência em relação ao traumatismo dentário (CALDERA et al., 2018; MORI et al., 2005; CHANDUKUTTY et al., 2017).

Neste trabalho, $32.9 \%$ dos participantes da pesquisa procurariam imediatamente o cirurgião dentista. Este índice foi semelhante ao trabalho de Chandukutty et al. (2017), o qual verificou que $23,4 \%$ da população estudada procuraria imediatamente o cirurgião dentista. Esses dados estão confrontantes aos encontrados por Mori et al. (2007), Alsadhan et al. (2018) e Kaul et al. (2017), os quais verificaram índices acima de $60 \%$. Vale ressaltar que $49,4 \%$ dos participantes da presente pesquisa acalmariam o acidentado e procurariam o pronto-socorro, e 3\% encaminhariam para hospitais; estes dados podem indicar que muitos dos participantes compreendem a importância do atendimento de urgência ao traumatismo dentário, e que os mesmos podem concluir que nestes ambientes há a presença de cirurgião dentista. Ao comparar as respostas entre os participantes que já tiveram experiência com traumatismo dentário e os não experientes, pode-se verificar ausência de diferença entre as condutas, ao contrário do que foi observado por Kaul et al. (2017). Cerca de $60 \%$ dos participantes da presente pesquisa relataram não ter sequelas ou as terem resolvido com tratamentos posteriores ao traumatismo; assim, acredita-se que isto pode ter influência na experiência, e que o traumatismo não promova grandes sequelas aos pacientes acometidos.

Em trabalhos realizados anteriormente, pode-se verificar que pequena parcela dos participantes fariam o reimplante imediato, com índices variando entre 5,8\% e 23,4\% (CHANDUKUTTY et al., 2017; KAUL, R. et al., 2017; CALDERA et al., 2018; ALSADHAN; ALSAYARI; ABUABAT, 2018; MORI et al., 2005). Estes dados estão de acordo com os encontrados na presente pesquisa. Sabe-se que o reimplante imediato é a conduta ideal para a avulsão dentária (ANDREASEN; ANDREASEN; ANDERSSON, 2018; ANDERSSON et al., 2012; TROPE et al., 2011), e campanhas de esclarecimento e orientação à população podem ampliar consideravelmente este ato, conforme verificado nos estudos de Arikan e Sonmez (2012) e Taranath et al. (2017). O mesmo pode ser observado em relação à limpeza do dente avulsionado com água corrente antes do reimplante dentário. A porcentagem mostrou-se reduzida $(20,3 \%$ das respostas deste pesquisa) à semelhança dos dados encontrados por Chandukutty et al. (2017) e Caldeira et al. (2018). Assim, campanhas de esclarecimento e orientação à população também devem incluir este aspecto.
Nos casos em que o reimplante dentário imediato não é realizado, a manutenção do dente avulsionado em meio de conservação adequado é fundamental (ANDREASEN; ANDREASEN; ANDERSSON, 2018; ANDERSSON et al., 2012; FLORES et al., 2016; MORI et al., 2010). O leite tem sido o meio de escoIha em virtude das suas propriedades de manutenção de viabilidade celular por até 6 horas, e por ser de fácil acesso (ANDREASEN; ANDREASEN; ANDERSSON, 2018; ANDERSSON et al., 2012; REIS et al., 2017; KHINDA, et al., 2017; MORI et al., 2010). Na presente pesquisa, este meio de conservação seria utilizado por somente $16 \%$ dos participantes. Em trabalhos anteriores (MORI et al., 2005; ALSADHAN; ALSAYARI; ABUABAT, 2018; KAUL et al., 2017; ARIKAN; SONMEZ, 2012; CHANDUKUTTY et al., 2017), a porcentagem para o uso do leite também foi reduzida, permanecendo entre $3,72 \%$ e $19,7 \%$. Nos casos em que o leite não é utilizado, pode-se optar pelo soro fisiológico ou pela saliva do paciente por um período máximo de $60 \mathrm{mi}$ nutos (ANDREASEN; ANDREASEN; ANDERSSON, 2018; ANDERSSON et al., 2012; KHINDA, et al., 2017; FLORES et al., 2016). Em pesquisas anteriores, a manutenção do dente avulsionado na boca do paciente em contato com a saliva foi reduzida (MORI et al., 2005; KAUL et al., 2017; CALDERA et al., 2018; ALSADHAN; ALSAYARI; ABUABAT, 2018) à semelhança do presente estudo. Apesar da possibilidade do uso da saliva, o não uso desta mostra-se favorável pela presença de contaminação na mesma, o que pode interferir negativamente na preservação celular (ANDREASEN; ANDREASEN; ANDERSSON, 2018; MORI et al., 2010). 0 soro fisiológico, apesar de ter osmolaridade diferente das células humanas (ANDREASEN; ANDREASEN; ANDERSSON, 2018; ANDERSSON et al., 2012; KHINDA, et al., 2017; FLORES et al., 2016) é menos prejudicial que o conservação do dente a seco, por exemplo, na mão do paciente ou embrulhado em gaze, papel, pano ou outros (ANDREASEN; ANDREASEN; ANDERSSON, 2018; ANDERSSON et al., 2012). No presente trabalho, o uso do meio a seco foi mais significativo que os demais, representando $43,7 \%$ dos relatos. Este percentual está de acordo com os encontrados nos trabalhos publicados por Mori et al. (2007) e Caldeira et al. (2018).

Arikan e Sonmez (2012) verificaram que uma campanha efetiva de esclarecimento da população ampliou o uso do leite como meio de conservação, posto que os percentuais de uso evoluíram de $10 \%$ antes da campanha para $86,6 \%$ após a mesma. 0 mesmo pode ser observado no trabalho de Mori et al. (2007). Estes dados confirmam que a realização de campa- 
nhas de esclarecimento à população é essencial para a ampliação dos índices de sucesso para o atendimento à avulsão dentária.

Ainda de acordo com os dados do presente trabalho, pode-se verificar que o tempo de trabalho na escola (16 anos ou mais) favoreceu o uso correto do meio de conservação para dentes avulsionados. Dados semelhantes foram encontrados por Kaul et al. (2017), o que pode demonstrar que o auxílio ao acidentado ou o compartilhamento de experiências amplie o conhecimento. $O$ grau de escolaridade não interferiu no conhecimento sobre o traumatismo dentário à semeIhança dos dados obtidos por Antunes et al. (2016). Os citados autores verificaram que somente $4,1 \%$ dos participantes de sua pesquisa tiveram orientações de primeiros socorros em relação ao traumatismo dentário durante a sua formação escolar. Esta justificativa pode ser projetada aos nossos resultados, evidenciando a ampla necessidade de campanha de esclarecimento sobre traumatismo dentário. 0 mesmo pode ser justificado para a ausência de diferença entre profissionais de escolas públicas e privadas.

É fundamental, também, estimular a população em geral sobre a importância da prevenção e do correto atendimento ao traumatismo dentário, destacando o quanto um acidentado pode ser beneficiado com estas condutas. Além disso, destaca-se a necessidade de inclusão deste aspecto de saúde pública no currículo escolar de formação de profissionais escolares.

Os professores e funcionários de escolas públicas e privadas apresentam reduzido conhecimento sobre traumatismo dentário, e as condutas desta população podem ser aprimoradas por meio de campanhas de esclarecimento.

\section{REFERÊNCIAS}

ALSADHAN, S. A.; ALSAYARI, N. F.; ABUABAT, M. F. Teachers' knowledge concerning dental trauma and its management in primary schools in Riyadh, Saudi Arabia. Int. Dent. J., v. 68, n. 5, p. 306-313, 2018.

ANDERSSON, L. et al. International Association of Dental Traumatology guidelines for the managementof traumatic dental injuries: 2. Avulsion of permanente teeth. Dent. Traumatol., v. 28, p. 88-96, Jan. 2012.

ANDREASEN, J. O.; ANDREASEN, F. M.; ANDERSSON, L. Textbook and color atlas of traumatic injuries to the teeth. 4th ed. Nova Jersey: Wiley-Blackwell, 2018.

ANTUNES, L. A. A. et al. Traumatic dental injury in permanent teeth: knowledge and management in a group of Brazilian school teachers. Dent. Traumatol., v. 32, p. 269-273, 2016.
ARIKAN, V.; SONMEZ, H. Knowledge level of primary school teachers regarding traumatic dental injuries and their emergency management before and after receiving and informative leaflet. Dent. Traumatol., v. 28, p. 101-107, 2012.

CALDERA, M. M. et al. Knowledge about emergency dental management among school teachers in Colombia: A baseline study to develop an education strategy. Dent. Traumatol., v. 34, p. 164-174, 2018.

CHANDUKUTTY, D. et al. Awareness of Dental Trauma Management Among School Teachers of Kannur District. J. Clin. and Diagn. Res., v. 11, n. 2, p. ZC08-ZC12, 2017.

DIANGELIS, A. J. et al. International Association of Dental Traumatology guidelines for the management of traumatic dental injuries: 1 . Fractures and luxations of permanet teeth. Dent. Traumatol., v. 28, n. 1, p. 2-12, 2012.

FLORES, F. W. et al. Meios de armazenamento para dentes avulsionados - uma revisão da literatura. Saúde, Santa Maria, Suppl, p. 73-80, jul. 2016.

GLENDOR, U. Epidemiology of traumatic dental injuries a 12 year review of the literature. Dent. Traumatol., v. 24, n. 6, p. 603-611, 2008.

KAUL, R. et al. Awareness of emergency dental trauma management among teachers of Kolkata. Ind. J. Dent. Res., v. 28, p. 595-603, 2017.

KHINDA, V. I. S. et al. Clinical and Practical Implications of Storage Media used for Tooth Avulsion. Int. J. Clin. Pediatr. Dent., v. 10, n. 2, p. 158-165, 2017.

MAGNO, M. B. et al. The relationship of previous dental trauma with new cases of dental trauma. A systematic review and meta-analysis. Dent. Traumatol., v. 35, n. 1, p. 3-4, 2019.

MORI, G. G. et al. Avulsion of permanent teeth: analysis of the efficacy of an informative campaign for professionals from elementary schools. J. Appl. Oral Sci., v. 15, n. 6, p. 534-538, 2007.

MORI, G. G. et al. Evaluating the knowledge of sports participants regarding dental emergency procedures. Dent. Traumatol., v. 25, n. 3, p. 305-308, 2009.

MORI, G. G. et al., Evaluation of the knowledge of tooth avulsion of school professionals from Adamantina, São Paulo, Brazil. Dent. Traumatol., v. 21, p. 1-4, 2005.

MORI, G. G. et al. Propolis as storage media for avulsed teeth: microscopic and morphometric analysis in rats. Dent. Traumatol., v. 26, n. 1, p. 80-55, 2010.

REIS, M. V. P. et al. Effect of different storage media on root dentine composition and viability of fibroblasts evaluated by several assay methods. Int. Endod. J., v. 50, n. 12, p. 1.185-1.191, 2017.

SANABE, M. E. et al. Urgências em traumatismos dentários: classificação, características e procedimentos. Rev. Paul. Pediatr., v. 27, n. 4, p. 447-451, 2009.

SKEIE, M. S.; AUDESTAD, E.; BARDSEN, A. Traumatic dental injuries - knowledge and awareness among present and prospective teachers in selected urban and rural areas of Norway. Dent. Traumatol., v. 26, p. 243-247, 2010. 
TARANATH, M.; SENAIKARASI, R. M.; MANCHANDA, K. Assessment of knowledge and attitude before and after a health education program in East Madurai primary school teachers with regard to emergency management of avulsed teeth. J. Indian. Soc. Pedod. Prev. Dent., v. 35, p. 63-67, 2017.

TROPE, M. et al. Avulsion of permanent teeth: theory to practice. Dent. Traumatol., v. 27, p. 281-294, 2011.

TROPE, M.; FRIEDMAN, S. Periodontal healing of replanted dog teeth stored in Viaspan, milk and Hank's balanced salt solution. Endod. Dent. Traumatol., v. 8, p. 183-188, 1992.

YAMASHITA, F. C. et al. Retrospective study on sequelae in traumatized permanent teeth. Eur. J. Dent., v. 11, n. 3, p. 275-280, Jul./Sept. 2017. 


\section{FIGURAS}

Figura 1 - Questionário Validado

\section{UNIVERSIDADE DO OESTE PAULISTA}

Faculdade de Odentologia

Titulo do Projeto: Avaliação do conhecimento e da conduta de urgência pós traumatismo dentário.

Atenção: para as questões abaixo, assinale somente uma opção.

Parte I - Questões sobre conhecimento gerais

1. Sexo:
( ) Masculino
( ) Feminino

2. Idade:
( ) 18 ou menos
( ) $41-60$
( ) $19-40$
( ) 61 ou mais

3. Educação:

( ) Ensino Fundamental ( ) Ensino Médio ( ) Ensino Superior

4. Tempo de trabalho na escola:
( ) $<1$ ano
( ) $11-15$ anos
( ) $1-5$ anos
( ) 16 ou mais
( ) 6-10 anos

\section{Parte II}

1. O que você faria se presenciasse algum tipo de traumatismo dentário (acidente envolvendo quebra do dente, corte no lábio, deslocamento do dente do local de origem)?

( ) Levaria o acidentado imediatamente para o dentista

( ) Acalmaria o acidentado e procuraria um pronto socorro

( ) Não saberia como proceder

( ) Procuraria um médico

( ) Iria para o hospital 
( ) Outro

2. Você já sofreu algum tipo de traumatismo dentário?

( ) $\operatorname{Sim}$ ( )Não

(Se sim, responder as questões de 3 a 5 , se não, responder a partir parte III)

3. Que tipo de lesão você teve?

( ) Fraturou a coroa do dente (parte visível do dente)

( ) Fraturou a raiz do dente

( ) $\mathrm{O}$ dente estava solto/mole mas não caiu da boca

( ) $\mathrm{O}$ dente caiu da boca completamente

( ) Fraturou algum osso do rosto

( ) Corte no lábio

( ) Outros

4. Como você procedeu?

( ) Não fez nada

( ) Procurou um dentista imediatamente

( ) Procurou um dentista no dia seguinte

( ) Foi para um pronto socorro

( ) Procurou serviços odontológicos universitários

( ) Outro

\section{Você teve alguma sequela?}

( ) Não

( ) Sim, porém fui submetido a tratamento e está tudo bem

( ) Sim, o dente teve que ser extraído

( ) $\mathrm{Sim}$, o dente mudou de cor

( ) Outro

\section{Parte III}

1. Você já teve alguma experiência com avulsão dentária (quando o dente sai por inteiro da boca)? Seja com você mesmo ou pessoa próxima. 


\section{Parte III}

1. Você já teve alguma experiência com avulsão dentária (quando o dente sai por inteiro da boca)? Seja com você mesmo ou pessoa próxima.

( ) $\operatorname{Sim}($ ) Não

2. Nos casos de avulsão dentária, você acha importante tomar alguma atitude com urgência?

( ) $\operatorname{Sim} \quad$ ( ) Não

3. Você mesmo (a) reimplantaria o dente avulsionado, ou seja, colocaria o dente de volta no seu local de origem?

( ) $\operatorname{Sim}$ ( ) Não

4. Você ach a necessário limpar o dente antes do reimplan te?

( ) $\operatorname{Sim} \quad$ ( ) Não

5. Se sim, o que faria?

( ) Lavar o dente com uma escova de dente

( ) Lavar o dente com água de tomeira

( ) Lavaria com Antisséptico (Ex.: Colgate, Listerine e outros)

( ) Desinfetaria o dente com álcool

( ) Nẫo faria nada

( ) Não sei

( ) Outros

6. Se não reimplan tasse o dente, como transportaria para o dentista?
( ) Na mão
( ) Enrolado em um papel, plástico ou pano
( ) Em um recipiente com água de torneira
( ) Em um recipiente com Leite
( ) Na boca do paciente
( ) Solução salina (ảgua salina ou soro fisiológico)
( ) Outros 
Figura 2 - Prevalência (dados expressos em porcentagens) do tipo de traumatismo dentário entre os professores e funcionários de escolas públicas e privadas

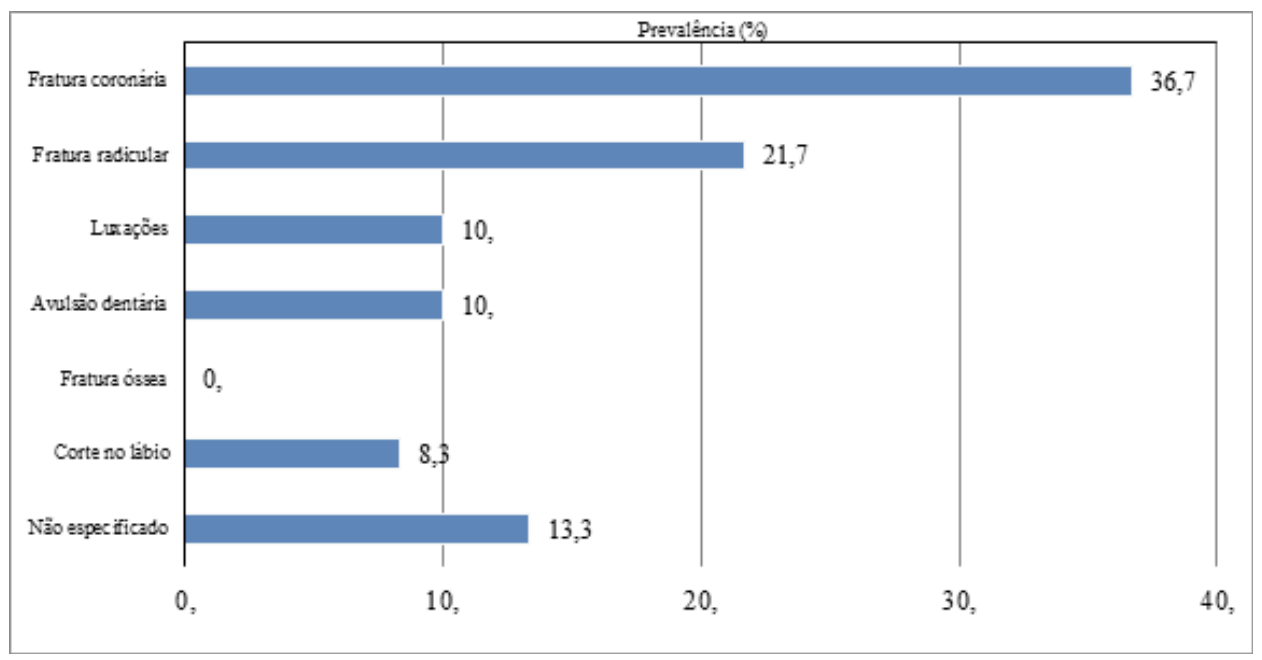

Figura 3 - Procedimentos referentes à limpeza a ser realizada nos dentes avulsionados antes do reimplante dentário (dados expressos em porcentagens)

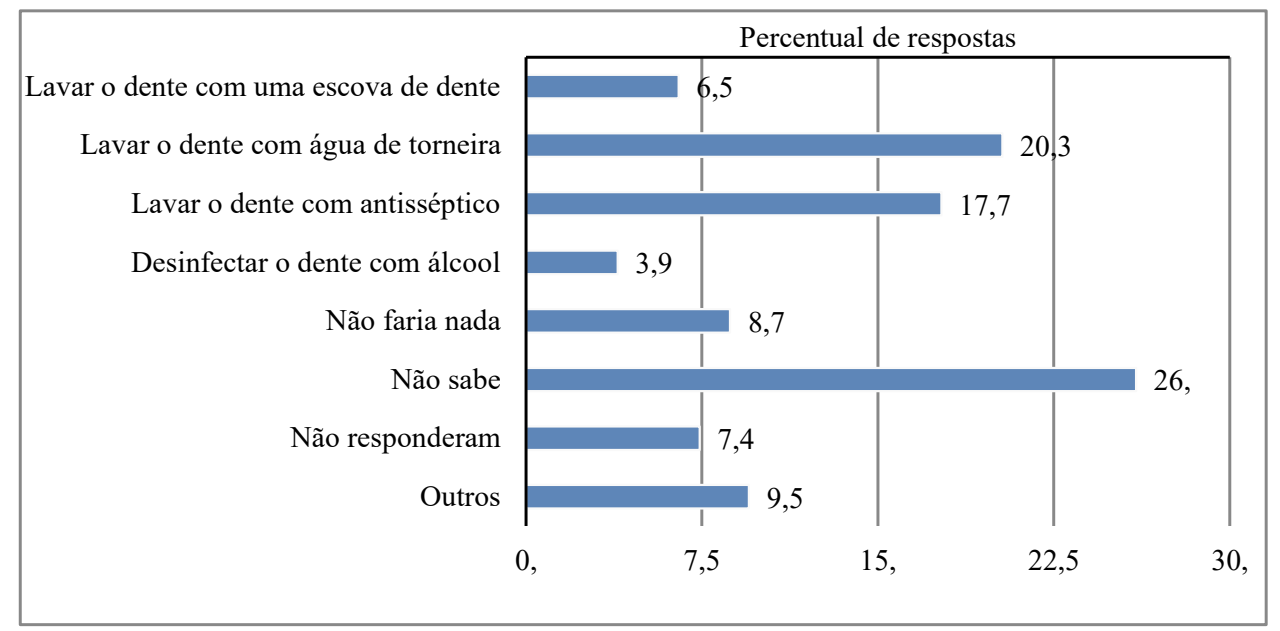

Figura 4 - Meios de conservação do dente avulsionado para transporte até o atendimento odontológico (dados expressos em porcentagens)

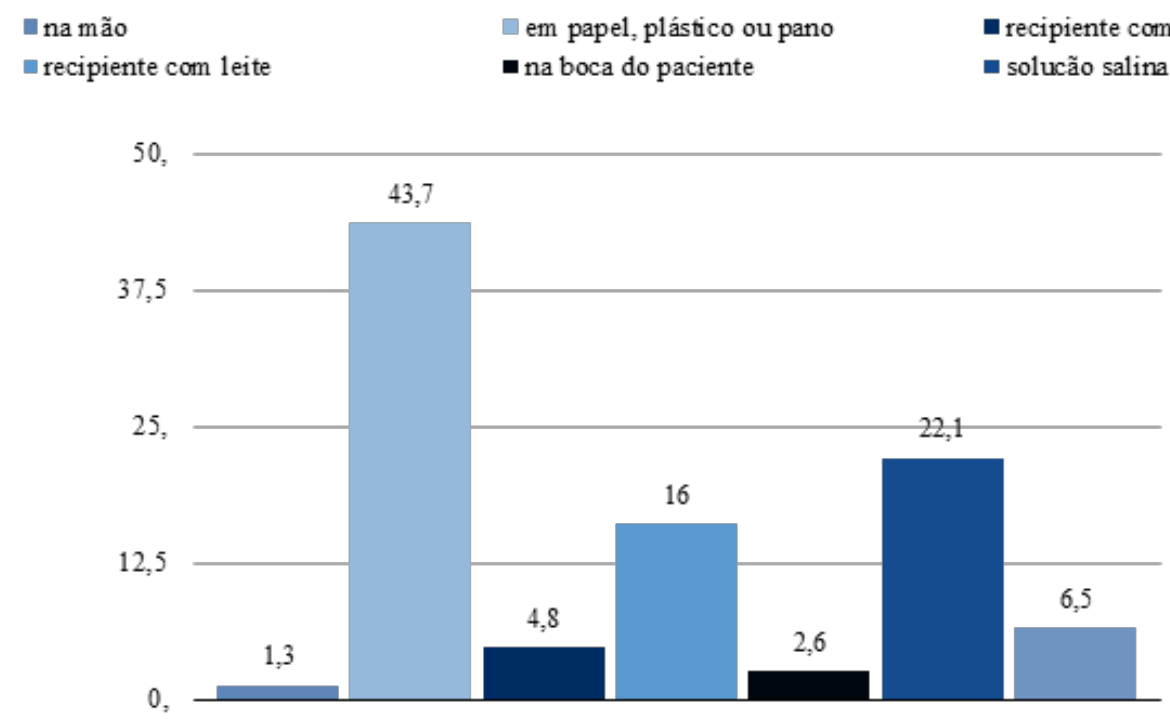

